\title{
Drivers' safety needs, behavioural adaptations and acceptance of new driving support systems
}

\author{
Farida Saad ${ }^{\mathrm{a}, *}$ and Pierre Van Elslande ${ }^{\mathrm{b}}$ \\ aFSTTAR-GRETTIA, 23, rue Alfred Nobel, Champs sur Marne, 77447 Marne la Vallée cedex 2, France \\ bIFSTTAR-MA, Chemin de la Croix Blanche, 13300 Salon-de-Provence, France
}

\begin{abstract}
The aim of this paper is to discuss the contribution of two complementary approaches for designing and evaluating new driver support systems likely to improve the operation and safety of the road traffic system. The first approach is based on detailed analyses of traffic crashes so as to estimate drivers' needs for assistance and the situational constraints that safety functions should address to be efficient. The second approach is based on in depth-analyses of behavioral adaptations induced by the usage of new driver support systems in regular driving situations and on drivers' acceptance of the assistance provided by the systems.
\end{abstract}

Keywords: Safety functions, Drivers needs, Accident study, Behavioral adaptation, Acceptance

\section{Introduction}

Over the past 20 years major technological changes have taken place in the automotive field. Many Research and Development programs (in Europe, USA and Japan) have been devoted to the design of new driver support systems (for route planning, obstacle detection, car-following situations, speed control, and so on). The development of these systems raises several theoretical and methodological questions. For psychologists and ergonomists the main issues can be stated as follows:

- What type of support could be appropriate for an activity such as driving, a complex activity performed in extremely diverse conditions by a highly heterogeneous population, both in terms of car usage (professional or private use, daily or occasional, etc.) and individual characteristics (age, experience, driving style, etc.)?

- Will the functions ascribed by the designers be compatible with drivers' needs, objectives and priorities when driving?
- How to assess the relevance of the support systems proposed; how to foresee their effects on drivers' behavior and their impact on traffic system operations and safety?

The relevance of the choices made to "help" the drivers and the predictions regarding the impact of the support systems largely depend on our knowledge of how the traffic system functions (and malfunctions), on the variables (situational and/or individual) likely to account for drivers behavior, and on the factors likely to explain the occurrence of traffic accidents. In other words it depends on the models (explicit or implicit) being referred to, traffic system and task models as well as models of drivers' activity or models of the individual "to be supported".

The aim of this paper is to discuss the contribution of two complementary approaches for dealing with such issues, with particular emphasis put on the safety impact of ITS (Intelligent Transport Systems) functions:

- Are they adequately defined for taking into account the real difficulties met by drivers? 
- $\quad$ Are they able to fit the situational constraints found in real traffic accidents?

- Will they receive a sufficient level of acceptance from drivers to be used "properly" or "as expected" and to fulfill their "safety functions"?

The first approach is based on detailed analyses of traffic crashes so as to estimate drivers' needs for assistance and the situational constraints that safety functions should address to be efficient.

The second approach is based on in depth-analyses of behavioral adaptations induced by the usage of new driver support systems in regular driving situations and on drivers' acceptance of the assistance provided by the systems. In both approaches, analyses are based on the identification of "critical" road events and scenarios and on the specification of operational criteria likely to assess and/or foresee the efficacy, efficiency and acceptance of the assistance provided.

\section{Drivers' needs to meet and contextual constraints to cope with}

Driving is a complex activity, and one of the main components of this complexity consists for the road user in the necessity to permanently share and control his/her limited attention resources at the right places and the right moments. This also involves keeping available a part of these resources in case of unexpected events, and to spare them in order to be able to function efficiently at the long term. Thus, every component of information added to the driving task is potentially able to consume attention capacity and maybe to lessen performance by leading to different forms of attention disturbances [1]. For that reason, ITS functions must be restricted to the drivers' needs in order not to overload or disturb their capacity: every technical system aimed at helping an operator has to be thought at the light of the real difficulties encountered by this operator. Whatever the devices, they should be defined so as to be valid, effective and acceptable for their users.

Traffic crashes attest that human functional capacity is sometimes exceeded in compensating for driving demands. As far as drivers are not willing to have them, every crash goes through a failure in one or another regulating function that usually enable them to compensate for the difficulties met at the wheel. Consequently, one way to get knowledge on the drivers needs in safety is to analyze these human function failures, their factors and the characteristics of the situations in which they occur. In this purpose, indepth accident studies make it possible to put forward these operating malfunctions, in relation to both the situational driving context (interaction with the vehicle, the road and with other road users) and the internal driving context (status, intentions, motivations, etc.) [2]. A previous study conducted in the frame of the European TRACE project made use of such accident data to evaluate the capacity of safety functions 1) to fulfill drivers' needs in safety, 2) to compensate for the contextual constraints found in accident situations [3]. The method used, which is illustrated in Figure 1, is also currently being applied in the frame of the actual European DaCoTa project so as to analyze more safety systems in that way.

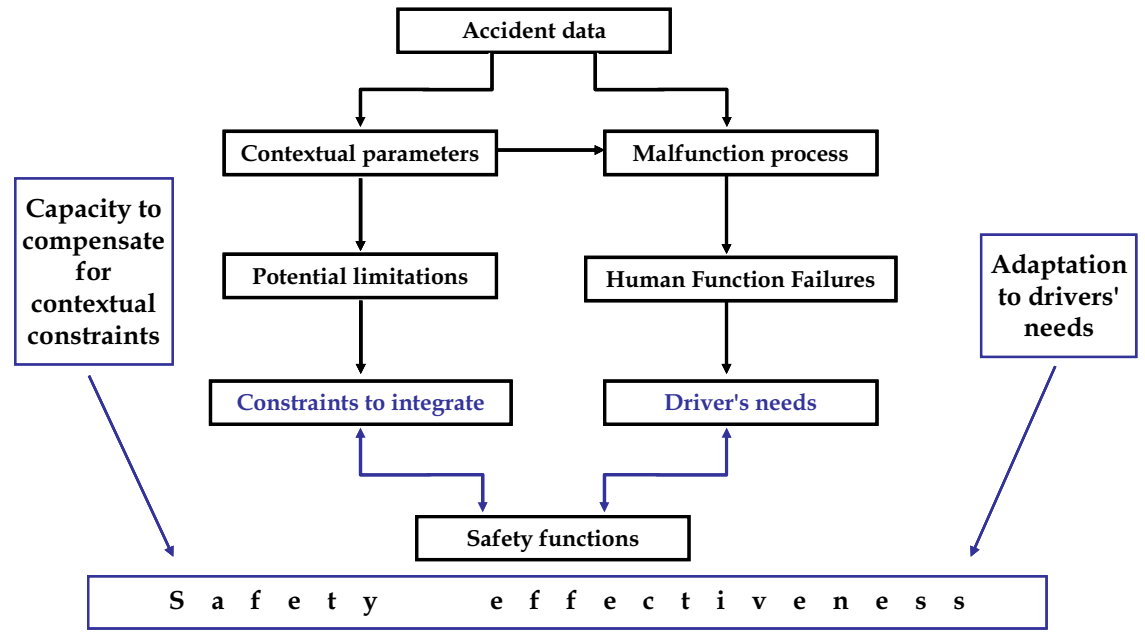

Fig. 1.Methodological chart 


\subsection{From human failures to drivers' needs}

From a systemic conception, a driver's safety need refers to something lacking inside the driving system functioning, in its defenses and/or in its protections [4]. Accidents are the symptoms of these lacks, and human functional failures are a more precise sign of what was lacking to the driver in order to compensate for the difficulties he met on the road. Consequently, a driver's need can be considered as the "negative" (the mirror) of a functional failure experienced by a driver when being unable to compensate for a difficulty met at the wheel: the need represents what would have avoided the failure if it had been fulfilled [5]. The in-depth study relying on 432 accident cases has allowed to put forward the main drivers needs as they could be inferred from the functional failures diagnosed (Table 1).

Table 1

Drivers pivotal needs distribution $(n=432)$ [6]

\begin{tabular}{|l|c|}
\multicolumn{1}{|c|}{ Driver needs } & $\%$ \\
\hline B01- Diagnosing driver condition & $11,8 \%$ \\
\hline B02- Diagnosing vehicle condition & $0,7 \%$ \\
\hline B03- Detecting an unexpected road difficulty & $2,2 \%$ \\
\hline B04- Detecting a fixed obstacle on the road & $3,0 \%$ \\
\hline B05- Detecting a slowly moving obstacle & $9,0 \%$ \\
\hline B06- Detecting an oncoming user in one's lane & $5,1 \%$ \\
\hline B07- Detecting a user on an intersecting course & $14,7 \%$ \\
\hline B08- Detecting a user outside the field of vision & $3,6 \%$ \\
\hline B09- Detecting a user in the forward field of vision & $4,0 \%$ \\
\hline B10- Detecting deviation from the path & $5,5 \%$ \\
\hline B11- Adapting speed to the road - 1: road geometry & $4,9 \%$ \\
\hline B12- Adapting speed to road network - 2: legislation & $1,0 \%$ \\
\hline B13- Evaluating a catching up on a slower user & $0,5 \%$ \\
\hline B14- Estimating a collision course with another user & $1,1 \%$ \\
\hline B15- Assessing gaps when changing lanes & $0,5 \%$ \\
\hline B16 Assessing gaps when cutting across traffic & $2,8 \%$ \\
\hline B17 Predicting that another user will fail to stop & $6,9 \%$ \\
\hline B18 Predicting that another user will stop & $1,9 \%$ \\
\hline B19 Predicting the manoeuvre of another user & $3,4 \%$ \\
\hline B20 Predicting the appropriate manoeuvre & $5,6 \%$ \\
\hline B21 Controlling one's vehicle & $11,7 \%$ \\
\hline & $\mathbf{1 0 0 , 0} \%$ \\
\hline
\end{tabular}

\subsection{Capacity of safety functions to meet drivers needs}

Driver's needs were then confronted with 21 primary safety functions, in order to estimate the ability of these functions to compensate for the needs according accident contextual constraints. The results obtained on a case by case basis gave an indication on the capacity of each function to address drivers' needs.

The overall result gives an optimistic figure of $85 \%$ of drivers' needs potentially met by the 21 functions considered as a whole. This result can be regarded as an "ideal" representing the potentiality of the systems if they were working in a perfect world. But in the accident world, it can be met possibly lowering parameters that have to be taken into account. Therefore a third step of analysis has been performed in order to define the contextual constraints found in accident reality, whereas they deal with human parameters (behavior, attitude, etc.) or with external limitations (space, time, velocity, etc.), which could lessen the ideal performance of each function regarding their ability to compensate for drivers' difficulties.

\subsection{Capacity of safety functions to compensate for contextual constraints}

The purpose of this step of analysis was to show the conditions under which the safety functions studied could compensate more efficiently for the difficulties that drivers found in context. Then have been analyzed, function by function, their potential capacity to compensate for these constraints, considering their specifications. The results show the potential drawbacks and weaknesses of each function when confronted with actual accident contexts. They allow, in turn, defining the parameters that these functions should integrate in order to maximize their safety benefit.

By integrating 1) adaptation to drivers' needs and 2) the capacity to compensate for accident contextual constraints, the study established the potential safety effectiveness of each function. An overall result gives a figure of $52 \%$ of capacity of these functions to compensate for drivers' needs. The detailed results gave indications on the parameters to integrate for improving the potential safety effectiveness of the functions studied as regard to drivers needs found in accidents [3].

But such an analysis turned toward prospective ergonomics must not be limited to the diagnosis of 
what would be needed from an accident research point of view. It is also necessary to take into account the more regular situations to which drivers are confronted and to examine how these new support systems will be used and accepted by drivers.

\section{In-depth analyses of behavioral adaptations and of drivers' acceptance}

In recent years, a variety of driver support systems have been designed with the objective of improving road safety such as Collision Avoidance System (CAS), Lane Departure Warning System (LDWS) or Speed Limiters (ISA).

While the crucial issues at a technical level have been mostly solved, their consequences on driver activity remain open and to be fully explained. In particular, the conditions of use of these new systems, their effects on driver behavior and strategies, and their impact on the operation and safety of the traffic system are of paramount concern amongst researchers and safety analysts.

\subsection{Behavioral adaptation in road safety research}

Amongst these issues, "Behavioral adaptations" have received particular attention. In road safety research, the term "Behavioral Adaptation" is mainly associated with "unintended or unexpected behavioral changes" that may appear in response to the introduction of a change in the traffic system and which may (more or less) jeopardize its expected safety benefits [7]. For example, in many research studies, it is assumed that driving with systems, which take over some elements of the driving task (such as speed and time headway control), may reduce drivers' workload and provide them with an opportunity for devoting less attention to the driving task. Another major concern is about drivers' ability to cope with the limitation of the support systems and to resume control in critical traffic scenarios. In some studies, particular emphasis is placed on the possible deterioration of drivers' interactions with other road users.

Different elements are assumed to influence the occurrence of behavioral adaptation such as the nature and the "perceptibility" of the changes introduced in the traffic system, (changes that directly influence the way the driving task is performed or changes that alter the driver's subjective safety, for instance), the degree of freedom that the change allows drivers (changes that give the driver an oppor- tunity for adapting his behavior) or the presence of competitive motives (safety versus mobility or productivity motives, for instance).

Although behavioral adaptation is a widely acknowledged phenomenon, the factors likely to explain it and the processes underlying its occurrence are not clearly established. Numerous processes may in fact come into play between the introduction of an "innovation" in the traffic system and its "adoption" by drivers, its "translation" into behavior (whether "safe" or "risky"), and its longer-term consequences on the operation and safety of the traffic system [8].

These processes should be analyzed in-depth and help to formulate hypotheses about the behavioral changes that may occur when using the new support systems, identify the conditions in which a "negative" compensation for safety might appear and direct thoughts on the means for preventing the occurrence or minimizing the extent of such negative changes.

\subsection{Studying the integration of new driver support system in driving activity and drivers' acceptance of the assistance provided}

Studying the integration of a new system into driving activity and identifying induced behavioral changes entail the consideration of several aspects [9] associated primarily to:

- The essential dimensions of road and traffic environments, such as: the number of interactions at play (between the driver, the vehicle, the infrastructure, and other road users) and their functional relationships, the regulatory, structural and dynamic demands of various road situations.

- The indicators for revealing the changes likely to take place in driver's activity.

Many empirical studies have been carried out, either in the "controlled" context of driving simulator or in the complexity of real driving situations, and some critical issues have already been identified as regards the usage and acceptance of the assistance provided and associated to two crucial peculiarities of the traffic system, i.e. the diversity of road and traffic situations and the diversity of the driver population. These issues are briefly described and discussed below (for a more detailed review see for example [10].)

\subsection{Diversity of road situations}

Many systems are designed to support drivers in maintaining some safety thresholds or ensuring com- 
pliance with some formal driving rules, such as adhering to legal speed limits, independently of the characteristics of road situations (infrastructure and traffic related) and the task being performed or planned, which determine drivers' control strategies.

Several studies reveal the influence of the road infrastructure and traffic conditions on the decision to use support systems or to follow their recommendations as well as on the magnitude of the behavioural changes observed when using them. They reveal that drivers' use and acceptance of new systems closely depends on the way they integrate (safety) formal rules in their driving and the tolerances they deem admissible.

Drivers' use and acceptance of the assistance provided also depend on the way they usually manage their interactions with other drivers. In many interaction situations drivers are reluctant to use the systems when doing so would require a significant deviation from their usual strategies. For example, drivers are prone to disengage a speed limiter in areas where speeding is the "norm" for the surrounding traffic and when they felt under pressure from other drivers.

These studies highlight the circumstantial requirements of driving assistance according to the dynamics of various driving situations and to the drivers' motives, objectives and intentions in these situations. They also confirm the need to adopt a multi level approach when assessing behavioural adaptations, that is to say to study possible changes within the activity of "assisted" drivers as well as within their interactions with other road users.

\subsection{Diversity of the driver population and potential differential impact}

Because of the great diversity of the driver population (both in terms of car usage and individual characteristics), many driver characteristics may be considered as relevant when studying usage and acceptance of new support systems, such as driver's age and gender, degree of experience and practice, and so on.

In some studies, the concept of "driving style" has received particular attention. Examples of dimensions of "driving styles" are driver's propensity to drive faster or slower than the surrounding traffic or to adopt short time headway in car-following situations. These studies suggest that driving styles play a role in the overall frequency of support system usage, in the magnitude of behavioral changes observed as well as in the acceptance of the assistance provided.
Other studies put the emphasis on some general personality traits, such as "Sensation Seeking" or "Locus of Control". These personality traits are assumed to influence, more or less directly, the occurrence of (negative) behavioral adaptation either through a general tendency for risk compensation or a propensity to manifest over-reliance in automation [11].

\subsection{Several issues for further research}

Briefly summarized the results obtained so far highlighted the difficulties of integration of new support systems in drivers' activity linked to an overly normative conception of assistance and to the substantial discrepancies between the functions assigned to these systems and drivers' objectives and strategies, depending on the situational context as well as on the characteristics of the drivers.

Furthermore, the results sometimes reveal an inverse relationship between effectiveness of the systems and their acceptability to drivers [11].

Such results raise important issues that deserve further research in our view:

- How to take into account the situational context when providing assistance to drivers?

- Could adapting the type of assistance provided to the context improve drivers' acceptance of the system?

- What degree of adaptability would nevertheless be appropriate in road safety terms?

- How can the right balance be found between safety effectiveness and drivers' acceptance?

\section{Conclusion}

The research work described in this paper aims at contributing to "safety-oriented prospective ergonomics" in the automotive field, and especially in the design and evaluation of new means for supporting drivers' activity and improving road safety.

Two complementary approaches were used in order to get a deeper understanding of the difficulties encountered by road users and to specify conditions and criteria likely to improve the efficacy and acceptance of safety oriented support systems.

A number of critical issues have been identified which deserve further research especially with the issue of finding the optimal compromise between safety effectiveness on the one hand and drivers' needs and acceptance on the other hand. 


\section{References}

[1] J. Hoel, M. Jaffard, C. Boujon and P. Van Elslande, Different forms of attentional disturbances involved in driving accidents IET Intelligent Transport Systems, 5 (2011), 120-126.

[2] P. Van Elslande and C.Nachtergaële, Human functioning and road accidents: a contribution to the study of potential limitations when taking informative driving aids into account. Recherche-Transports-Sécurité, English issue, 9 (1993), 27-36.

[3] P. Van Elslande, V. Vatonne, H. Vallet, K. Fouquet, B. Canu and J-Y. Fournier, Assessing drivers' needs and contextual constraints for safety functions: A human centered approach from in-depth accident analysis. European TRACE project, Deliverable 4.1.5, 2008.

[4] S. W. A. Dekker, Reconstructing human contributions to accidents: the new view on human error and performance, Journal of Safety Research, 33 (2002), 371-385.

[5] G. Malaterre, H. Fontaine and P. Van Elslande, Analysis of driver needs using accident reports: an a priori evaluation of Prometheus functions. Arcueil: INRETS, Research report $\mathrm{n}^{\circ} 139,1992$.

[6] P. Van Elslande, P, What needs and constraints for safety functions? A human-centered investigation from in-depth accident data. Annals of Advances in Automotive Medicine, 53, 2009
[7] OECD. Behavioural Adaptations to changes in the road transport system. Organization for Economic Co-operation and Development, Paris, 1990.

[8] I.D. Brown. Concepts and definitions in road safety. In M.B. Biecheler, C. Lacombe and N. Mulhrad (Eds), Evaluation 85, proceedings of the international Meeting on the Evaluation of Local Traffic Safety Measures (413-422), Paris, France, 1985.

[9] F. Saad and T. Villame, Intégration d'un nouveau système d'assistance dans l'activité des conducteurs d'automobile. In J-M Ganascia (Ed), Sécurité et Cognition (105-114). Paris: Edition Hermes, 1999.

[10]F. Saad, Some critical issues when studying Behavioural Adaptations to new driver support systems. Cognition, Technology \& Work, 8 (2006), 175-181.

[11]B.A. Jonah, R. Thiessen, R. and E. Au-Yeung, E. Sensation seeking, risky driving and behavioral adaptation. Accident Analysis and Prevention, 33 (2001). 679-684.

[12] S. Lassarre and F. Saad. An Integrated and Multidisciplinary Approach for Studying Use and Acceptance of New Driver Support System: The French National Project on Intelligent Speed Adaptation (LAVIA Project). ICTIS 2011: Multimoda Approach to Sustained Transportation System DevelopmentInformation, Technology, Implementation Volume I: Highway Transportation ( 1971-1978), 2011 